Artículo de Investigación

\title{
Evaluación de la movilidad de estudiantes y accesibilidad espacial a centros de educación en zonas periurbanas
}

\author{
Evaluation of student mobility and spatial accessibility to educational centers in peri-urban areas

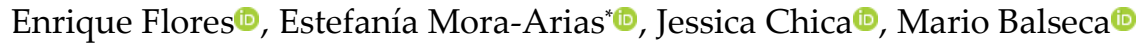 \\ ${ }^{1}$ Grupo de Investigación CITMOV, Facultad de Arquitectura y Urbanismo, Universidad de Cuenca, Cuenca, Ecuador; 0101168; \\ enrique.flores@ucuenca.edu.ec; jessica.chica@ucuenca.edu.ec; maboca11@gmail.com
}

*Correspondencia: estefania.mora@ucuenca.edu.ec

\begin{abstract}
Citación: Flore, E., Mora-Arias, E., Chica, J., \& Balseca, M., (2022). Evaluación de la movilidad de estudiantes y accesibilidad espacial a centros de educación en zonas periurbanas. Novasinergia. 5(1). 128149.

https://doi.org/10.37135/ns.01.09.08
\end{abstract}

Recibido: 09 noviembre 2021

Aceptado: 29 enero 2022

Publicado: 31 enero 2022

Novasinergia

ISSN: 2631-2654

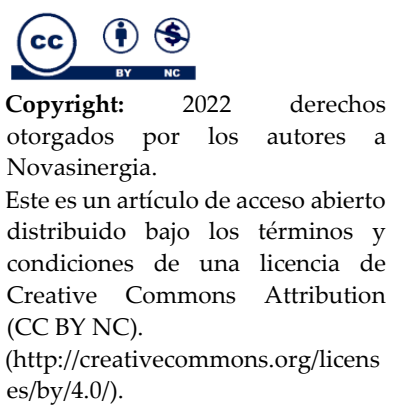
es/by/4.0/)
Resumen: La educación es condición esencial para alcanzar el desarrollo de las sociedades, por ello es necesario que se garantice su accesibilidad a toda la población y especialmente a los sectores desfavorecidos. En este trabajo se analizó la accesibilidad espacial de la población a los centros educativos de niveles de enseñanza primaria y secundaria de carácter público en cuatro parroquias periurbanas del cantón Cuenca. Se plantearon dos enfoques para el tratamiento de datos, el primero a nivel de hogar del cual se obtuvo información de 408 estudiantes, y una segunda a nivel de áreas consolidadas que formula escenarios de accesibilidad, relacionando costos de desplazamientos en términos de tiempo y distancia. Estos escenarios se modelaron a través de herramientas basadas en Sistemas de Información Geográfica. Se determinó que el medio más utilizado es a pie con el $54,38 \%$ con un tiempo promedio de traslado de 13,5 minutos, lo que indica cercanía a los centros educativos a pesar de tratarse de áreas periurbanas.

Palabras clave: Accesibilidad, educación, periurbano, planificación, transporte.

Abstract: Education is an essential condition to achieve the development of societies; therefore, it is necessary to guarantee its accessibility to the entire population and specially to disadvantaged sectors. This study analyzed the spatial accessibility of the population to public primary and secondary education centers in four peri-urban parishes in the canton of Cuenca. Two approaches were used for data processing. The first one was at the household level. As a result, information was obtained for 408 students. The second one is at the level of consolidated areas that formulates accessibility scenarios, relating travel costs in time and distance. These scenarios were modeled using tools based on Geographic Information Systems. As a result, the most used means of transportation is by foot (54.38\%) and an average travel time of 13.5 minutes. Thus, the educational centers are close to each other even though they are peri-urban.

Keywords: Accessibility, education, peri-urban, planning, transportation. 


\section{Introducción}

En los últimos años las ciudades Latinoamericanas han sufrido un acelerado crecimiento reflejado en la expansión de grandes zonas metropolitanas (Hernandez et al., 2009). Dicho crecimiento implica la incorporación de extensiones considerables de suelos agrícolas como soporte de nuevas actividades urbanas (De Oliveira \& Hurtado, 2017). La conurbación resultante de este proceso ha derivado en una serie de transformaciones territoriales, con resultados heterogéneos de acuerdo con las particularidades de cada región, en donde los territorios de menor jerarquía se someten a los requerimientos de las ciudades y se integran a su dinámica bajo formas muy diversas (Hidayati, Tan \& Yamu, 2021).

El diagnóstico de la situación social y económica en las zonas periurbanas y rurales de América Latina presenta un panorama complejo, con varias necesidades insatisfechas entre ellas la educación en sus distintas formas y niveles (Gajardo, 2014). Según el informe de la Organización de las Naciones Unidas para la Educación, la Ciencia y la Cultura (UNESCO, 2013), los problemas de falta de equidad, mala calidad y bajo rendimiento de los sistemas escolares continúan afectando con fuerza a las poblaciones en situación de pobreza, que viven y trabajan en zonas rurales. La distribución desigual de las oportunidades educativas distingue a quienes acceden al sistema escolar pero no logran aprobar los primeros años de educación obligatoria; y los que logran adquirir conocimientos básicos de lectura, escritura y cálculo, pero abandonan sus estudios para incorporarse a la vida productiva (Terigi \& Shanno, 2013).

En este contexto, el transporte escolar se perfila como un instrumento de vital importancia, sobre todo en núcleos pequeños de población que no cuentan con una escuela, colegio o instituto cercano (Badía, 2016). Este tipo de transporte asegura que los estudiantes de medios rurales puedan ejercer en igualdad de condiciones su derecho a la educación (Raikes, Straw \& Linton, 2015), permitiendo el acceso a oportunidades de desarrollo de las que carecen en sus núcleos de origen.

Para el caso de Argentina, en las localidades de Maipú y General Guido, se determinó las distancias recorridas por los alumnos y los medios de transporte utilizados para sus desplazamientos. La mayoría llegan a la escuela en movilidad propia, son llevados por su familia en auto particular o incluso algunos se trasladan a caballo, o son llevados por su maestra para garantizar su continuidad en el sistema. El recorrido de los alumnos de la zona rural de Maipú es en promedio $6.79 \mathrm{~km}$, diarios; y el recorrido de los alumnos rurales de General Guido es en promedio $8.54 \mathrm{~km}$ diarios (Annessi, Bachex \& Demirta, 2018).

Escobar, Urazán \& Moncada (2017) reportaron que en la zona rural del departamento de Caldas en Colombia se ha analizado características de accesibilidad a diferentes equipamientos, definidos como nodos de actividad primaria, divididos en: seguridad, salud y educación. Cada nodo fue categorizado a través de elementos de accesibilidad, como infraestructura vial, donde se realizaron análisis geoestadísticos, de conectividad y curvas isócronas. Este estudio permitió definir, tanto la concentración de los nodos, como su conectividad, encontrando como resultado mayor volumen de traslado y en menor tiempo 
a universidades y al sistema de salud. Finalmente recomienda, elementos de infraestructura para medios de transporte sostenible, considerando una topografía accesible.

Desde otro enfoque, Alvarez, Quirós \& Gutiérrez (2019) analizaron la accesibilidad peatonal de la población en edad escolar entre 5 y 14 años, hacia centros educativos de enseñanza en niveles de básica primaria y secundaria en la ciudad de Ibagué en Colombia. Determinaron mediante procesos cuantitativos que, el $71 \%$ de la población posee un centro educativo a menos de 10 minutos de distancia. Seguido de un $23 \%$ que comprende tiempos entre 10 a 20 minutos caminables; finalmente, un $6 \%$ de accesibilidad baja que supera los 20 minutos de desplazamiento peatonal en 3 comunas ubicadas en la periferia de la ciudad. En resumen, los autores establecieron que, a pesar de observar que la accesibilidad espacial sobre el territorio es buena, la atención a la demanda escolar no lo es. Por tanto, los costes en los desplazamientos peatonales aumentan y dificultan el acceso al servicio por la necesidad de emplear más tiempo y medios motorizados para tal fin (Alvarez et al., 2019).

En un estudio realizado para el área rural de Concepción, Chile, De la Fuente, Rojas \& Salado (2013) modelaron diferentes zonas de influencia de 500 y $1000 \mathrm{~m}$ a centros educativos, es decir, se formaron franjas de accesibilidad vinculadas a unidades muestrales (manzanas censales) utilizando los siguientes criterios:

-A menos de $500 \mathrm{~m}$, distancia de proximidad peatonal ideal a los equipamientos (educación).

-Entre 500 a 1000 m se localizan manzanas censales accesibles.

-Mayor a 1000 m se encuentra la población sin cobertura, es decir fuera del umbral de análisis.

Al evaluar los resultados se determinó que el 76.46 \% de la población total en un rango de edad de 0 a 17 años se encuentra cubierta por el servicio educativo en torno a los principales anillos (de 500 a $1000 \mathrm{~m}$ del área de influencia).

En el marco nacional, el Ministerio de Educación del Ecuador, como ente rector de los procesos concernientes al desarrollo del sistema educativo, ha establecido políticas públicas referentes a infraestructura educativa tanto urbana como rural, considerando aspectos como: distancia máxima de accesibilidad de $500 \mathrm{~m}$; infraestructura adecuada para movilidad; un espacio seguro en lo que respecta al uso de suelo; y un tamaño adecuado de disposición (Ministerio de Educación, 2012). Si bien es cierto, a nivel nacional se establece parámetros de planificación urbanística para equipamientos educativos, a un nivel local, ciudades como Quito (capital del Ecuador) define en su normativa de uso de suelo áreas de influencia de equipamientos según su nivel de servicio, es decir, preescolares y escuelas un radio de influencia de hasta $400 \mathrm{~m}$, mientras que colegios y unidades educativas una distancia no mayor a 1000 m (Municipio del Distrito Metropolitano de Quito, 2003).

Decidir la ubicación de un centro educativo no es únicamente un problema de planificación de construcción; sino que está estrechamente relacionado con su accesibilidad. El acceso abarca muchos valores y prácticas, incluida la justicia, la equidad espacial y socia (Talen, 2001). Los planificadores y formuladores de políticas, necesitan información sobre el tamaño de la población para garantizar la accesibilidad a las instalaciones públicas (Jega, Comber \& 
Brusndon, 2012), en este sentido, en algunos países se consideran datos de la población en edad escolar con el fin de realizar análisis de distribución geográfica de los establecimientos y decidir sobre los tipos, tamaños y ubicaciones (Châu, 2003). Este tipo de análisis son fundamentales en países en vías de desarrollo, dado el rápido crecimiento de su población.

Para satisfacer esta necesidad, los sistemas de información geográfica (SIG) ofrecen métodos innovadores y precisos para contribuir a la planificación de infraestructura educativa (Köse, Koçyiğit, Erdem \& Jega, 2021). Estas herramientas establecen una mejor visión de la realidad al permitir georeferenciar, organizar y normalizar la información para una mayor efectividad en la toma de decisiones (Pueyo, 1991; Pino, Astudillo, Aguirre \& Salazar, 2019). En consecuencia, los SIG se pueden utilizar para planificar la ubicación de nuevos centros educativos en función de los datos relacionados con la población, las carreteras de la ciudad, la ubicación de los equipamientos existentes (Köse et al., 2021), entre otros.

Bajo este contexto, la presente investigación tiene como objetivo analizar la accesibilidad a los centros educativos en el área periurbana de Cuenca en Ecuador, partiendo de un análisis con dos enfoques: a nivel de hogar en el que se analiza el comportamiento de movilidad de los estudiantes hacia los centros educativos públicos y a nivel de áreas consolidadas (espacio-temporal), en el que se formulan escenarios de accesibilidad relacionando costos de desplazamientos en términos de tiempo y distancia. Estos escenarios se modelaron a través del empleo de SIG.

\section{Metodología}

\section{1. Área de estudio}

El cantón Cuenca, ubicado en la provincia del Azuay, es el tercero más importante del Ecuador, con una extensión territorial de $3665 \mathrm{~km}^{2}$, seccionada de manera política y administrativa en 15 parroquias urbanas y 21 rurales (GAD Municipal del Cantón Cuenca, 2015). La población de Cuenca para el año 2010 era de 524536 habitantes, mientras que en el 2020 la proyección es de 636996 habitantes (Instituto Nacional de Estadística y Censos, 2010a), lo que indica un crecimiento del $17.65 \%$.

Para definir las áreas de estudio se consideró lo establecido por el Plan de Desarrollo y Ordenamiento Territorial - PDOT- (GAD Municipal del Cantón Cuenca, 2015) vigente, se ha seleccionado como áreas de estudios a las parroquias rurales de Ricaurte, El Valle, Baños y Sinincay (asentamientos de jerarquía 2, puesto que la jerarquía 1 corresponde a la cabecera cantonal - ciudad de Cuenca), por ser considerados como nodos de desarrollo, por su ubicación geográfica a menos de $15 \mathrm{~km}$ de la ciudad de Cuenca, por ser las parroquias rurales más pobladas y contar con vías de acceso en buen estado (Figura 1). 


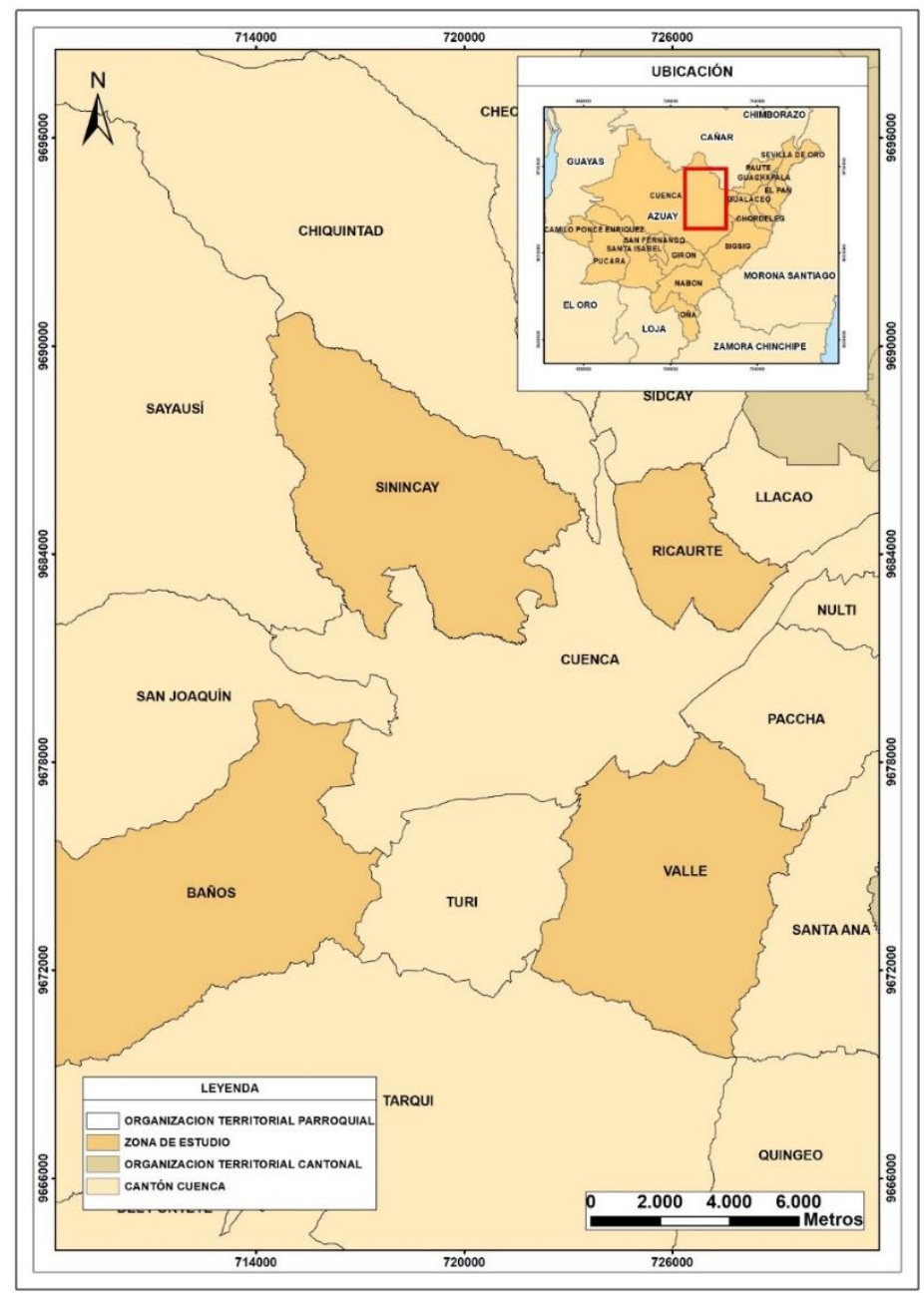

Figura 1: Localización de las áreas de estudio.

En las parroquias periurbanas de estudio existe una alta concentración de población infantil y joven (5-19 años) que representa un promedio del 32.75 \% de su población total (Instituto Nacional de Estadística y Censos, 2010a). Este indicador define la importancia para esta zona de la implementación adecuada de servicios e infraestructura educativa, los que deben cumplir con los requerimientos establecidos a través de las políticas públicas definidas a nivel nacional.

En lo que respecta a los niveles de educación de las parroquias de Baños, Sinincay, El Valle y Ricaurte para población sobre los 5 años, se ha determinado que el $81 \%$ cuenta con al menos un nivel básico de instrucción (Instituto Nacional de Estadística y Censos, 2010b), distribuido entre los diferentes niveles, siendo el primario el de mayor asistencia con una tasa del $40.8 \%$ (Instituto Nacional de Estadística y Censos, 2010c), esto refleja un acceso importante a los sistemas de educación del cantón. Sin embargo, se debe tomar en cuenta que, la infraestructura no suele tener una ubicación adecuada con respecto a los hogares de estas zonas, por lo que deben trasladarse al área urbana que cuenta con mayor número de instituciones. Es por esto que las políticas sobre accesibilidad e infraestructura educativa deben integrarse y fortalecerse, como un eje del bienestar de los habitantes de zonas con población en constante crecimiento. 


\subsection{Flujo de actividades}

Para analizar la accesibilidad de la población a los centros de educación públicos en las zonas periurbanas de Cuenca se utilizó una metodología cuantitativa con dos enfoques: a nivel de hogar, en el que a través de información primaria se analizó las condiciones de movilidad de los estudiantes, y a nivel de áreas consolidadas, en el que con un análisis espacio temporal se localizaron las zonas más consolidadas de las parroquias de estudio en la última década: 2010 - 2020 y con base en ello se formularon escenarios sobre la accesibilidad a los centros educativos identificados en el análisis a nivel de hogar. El proceso metodológico utilizado se detalla en la figura 2.

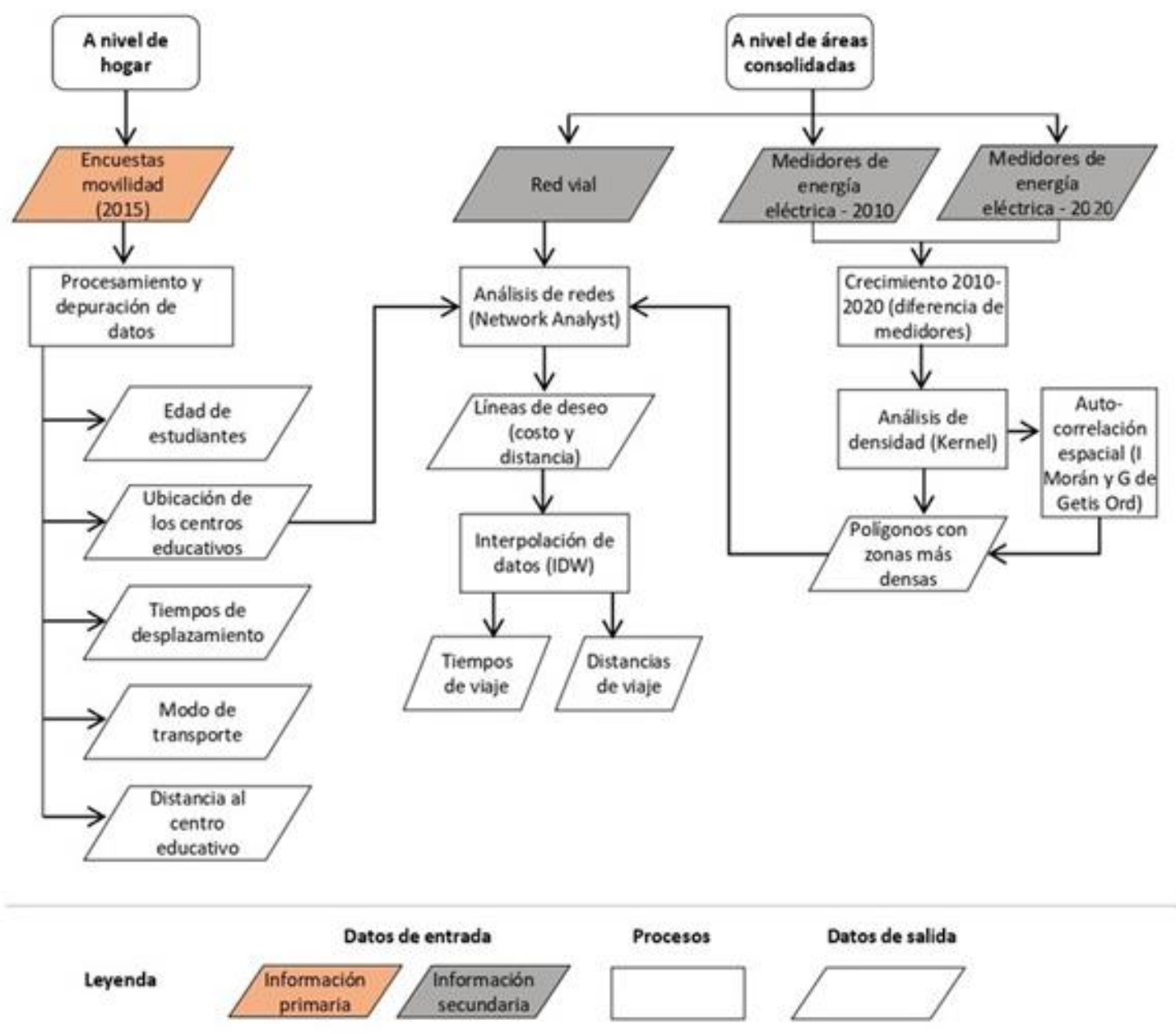

Figura 2: Flujo de actividades para el análisis de accesibilidad a centros educativos.

\subsection{Datos y variables}

Para el análisis a nivel de hogar se realizó una encuesta en el año 2015 enfocada principalmente en registrar información referente a los hábitos de movilidad cotidiana de cada uno de los miembros de las familias encuestadas. Se calculó una muestra con un índice de confiabilidad del $95 \%$, y un margen de error del $5 \%$. Estos porcentajes permiten una calidad de datos óptima con respecto al estudio que se va a realizar. Se registró un total de 1158 hogares en el que se obtuvo información de 1326 personas que viajan diariamente por motivo estudio. Para el análisis se excluyó los registros de estudios superiores (universidad 
o institutos) y aquellos que viajan a equipamientos de carácter particular. Se obtuvo información de 408 estudiantes y se analizaron cinco variables a nivel de hogar:

- Edad de los estudiantes: se definieron tres grupos de acuerdo con los siguientes niveles de escolaridad, de 3-5 años pertenecientes a educación inicial (preescolar), de 6-11 años en niveles de educación primaria (escuelas), y de 12-20 años alumnos que asisten a centros educativos de nivel secundario (unidades educativas, colegios). Para el análisis de los resultados el nivel preescolar se lo agrupa con el nivel de educación primaria dado que varios sistemas de educación a nivel de Latinoamérica lo conjugan de esta manera.

- Ubicación de las instituciones de educación pública: se realizó una referencia geográfica de los principales centros educativos de los estudiantes que respondieron la encuesta.

- Medio de transporte: esta variable describe los principales medios de transporte utilizados por los estudiantes para dirigirse hacia los centros educativos, siendo estos: a pie, en transporte público colectivo, vehículo de alquiler, vehículo propio, moto o bicicleta.

- Tiempo: representa el tiempo promedio empleado para el desplazamiento de los estudiantes hacia su centro educativo, medido en minutos.

- Distancia: este parámetro comprende el desplazamiento promedio (distancia euclidiana) de los estudiantes desde su lugar de origen (vivienda) hacia su destino (centro educativo), medido en metros.

En el análisis a nivel de áreas consolidadas se requirió información geoespacial de las viviendas en la zona de estudio y su variación multitemporal entre los años 2010 y 2020, no obstante, ante la carencia de este tipo de información se utilizó los datos proporcionados por la Empresa Eléctrica Regional Centro Sur, entidad que cuenta con una base especializada de los medidores clasificados por el año de implementación. Para efectos del estudio, se consideró que un medidor de luz representa una vivienda.

Por otro lado, la red vial juntamente en conjunto con valores de impedancia dentro de sus atributos es fundamental para formular modelos de accesibilidad. El cálculo de la impedancia, se realizó contemplando un valor medio de velocidad peatonal de $4.5 \mathrm{~km}$ (Ecuación (1)) (Muñoz-Raskin, 2010):

$$
I=\frac{60 \times L}{1000 \times V}
$$

En ecuación (1), I representa la Impedancia expresada en min, $L$ representa la distancia recorrida en $\mathrm{m}, V$ es la etiqueta para la velocidad promedio peatonal en $\mathrm{km} / \mathrm{h}$. Así, los modelos de accesibilidad se construyeron con datos obtenidos de la plataforma OpenStreetmap y ortofotos del área de estudio.

\subsection{Análisis de información}

Para el análisis a nivel de hogar se utilizó métodos descriptivos y de inferencia estadística, lo que permitió reducir el conjunto de datos obtenidos por un pequeño número de valores descriptivos. Este procedimiento permitió dilucidar las principales propiedades de los datos observados, así como las características clave de los fenómenos bajo 
investigación. Para el análisis a nivel de áreas consolidadas se definieron tres procesos: delimitación de las áreas consolidadas, análisis de redes y cálculo de tiempos y distancias de viaje.

Para la delimitación de las áreas consolidadas: se consideró la variación de la prestación de servicio eléctrico entre los años 2010 y 2020, un elemento esencial en el desarrollo del estudio es definir la existencia de una relación aparente de los datos (base de consolidación) como el grado de este dentro de un ámbito espacial. La autocorrelación espacial es un procedimiento que determina el nivel de asociación o agrupación de una variable en un ámbito geográfico definido. La clave de su resultado es el grado de asociación con el que cuentan los elementos espaciales que se van a analizar, dado que, una agrupación más compacta permitirá determinar de mejor manera los resultados (Siabato \& GuzmánManrique, 2019). La consideración del uso de esta herramienta en la investigación permite definir el grado de relación de viviendas desde una perspectiva espacial, y de accesibilidad. Adicionalmente, la aplicación del índice " $\mathrm{I}$ " de Moran junto con el índice "G" de Getis Ord, permiten identificar elementos de autocorrelación con respecto a sistemas de variables de empleo (Chaparro \& Hernández, 2020). Además, como parte esencial de esta investigación, se deben establecer densidades con respecto a las zonas de estudio, para lo cual se empleará la herramienta Kernel.

Como parte esencial de esta investigación, se deben establecer densidades con respecto a las zonas de estudio. El cálculo de densidades de Kernel permite identificar zonas de mayor densidad de puntos (mapa de calor), considerando estos datos como valores espaciales, es decir, que se ubican sobre un espacio y que su relación se caracteriza por la distancia entre ellos, generando valores de mayor cercanía y separación (Guzmán, 2020). Este método también se caracteriza por la asignación de pesos que se enlazan y posteriormente crean una asociación, lo que define una estimación adecuada (Puchades, 2017). La factibilidad de esta herramienta para la puntualización de la accesibilidad a instituciones educativas radica en determinar, como se ha generado una concentración de viviendas en base a los datos de los nuevos medidores implementados en las parroquias en estudio. Con estos datos se podrá definir las zonas con mayor concentración, para de esta manera determinar si los procesos de accesibilidad coinciden con la localización de las viviendas que presentan mayor densidad, además de la ubicación de las instituciones educativas con respecto a la misma.

Para el análisis de redes se parte de la determinación de los puntos de origen, es decir las áreas con mayor consolidación y los centros educativos como puntos de destino (Rodríguez, 2011). El modelo de análisis de redes se basa en la teoría de grafos que responde a un esquema lineal que sirve para precisar la estructura topológica de la red, con un encadenamiento de parámetros tales como la longitud, dirección y conectividad (Rodríguez, 2011).

Para el cálculo de tiempos y distancias de viaje, se procede con la técnica de IDW o también conocida como ponderación de la inversa de la distancia para medias móviles (Navarrete \& López, 2019). Se debe tener en cuenta la existencia de un ajuste del tiempo para determinar la ubicación entre diferentes puntos, considerando que no son elementos lineales, y que es necesario establecer o predecir datos intermedios para crear una ponderación adecuada (Nieto \& Márquez, 2018). Esta investigación requirió analizar valores de temporalidad 
además de la distancia, dado que se requería definir valores que permitan establecer una uniformidad con la accesibilidad desde los puntos de origen y destino, y como estos intervienen en la definición de valores.

\section{Resultados}

\subsection{Accesibilidad a nivel de hogar}

Los datos muestran que, en las áreas de estudio, el mayor porcentaje de estudiantes encuestados son mayores de 12 años, $68.03 \%$, lo que representa una demanda importante por centro de estudios secundarios. El $27.25 \%$ representan el grupo etario de 6 a 11 años, que corresponde a niños de escuela de nivel básico y finalmente, un porcentaje del $4.73 \%$ en un rango de 3 a 5 años. Todos estos son grupos etarios que requieren de centros educativos específicos correspondientes al sistema educativo. De la misma manera se consideran diferentes requerimientos de movilidad. En cuanto a la ubicación de los centros educativos públicos se registraron 26 destinos, entre escuelas, colegios y unidades educativas (nivel de educación primaria y secundaria) como se muestra en la figura 3. Esta información se utilizó posteriormente para generar modelos de accesibilidad.

En relación a la distribución espacial de los centros educativos, si bien la mayor cantidad se encuentra en las mismas parroquias analizadas (17 centros), también existen estudiantes que se desplazan fuera de su parroquia, ya sea en la ciudad de Cuenca o hacia otra parroquia rural (9 centros).

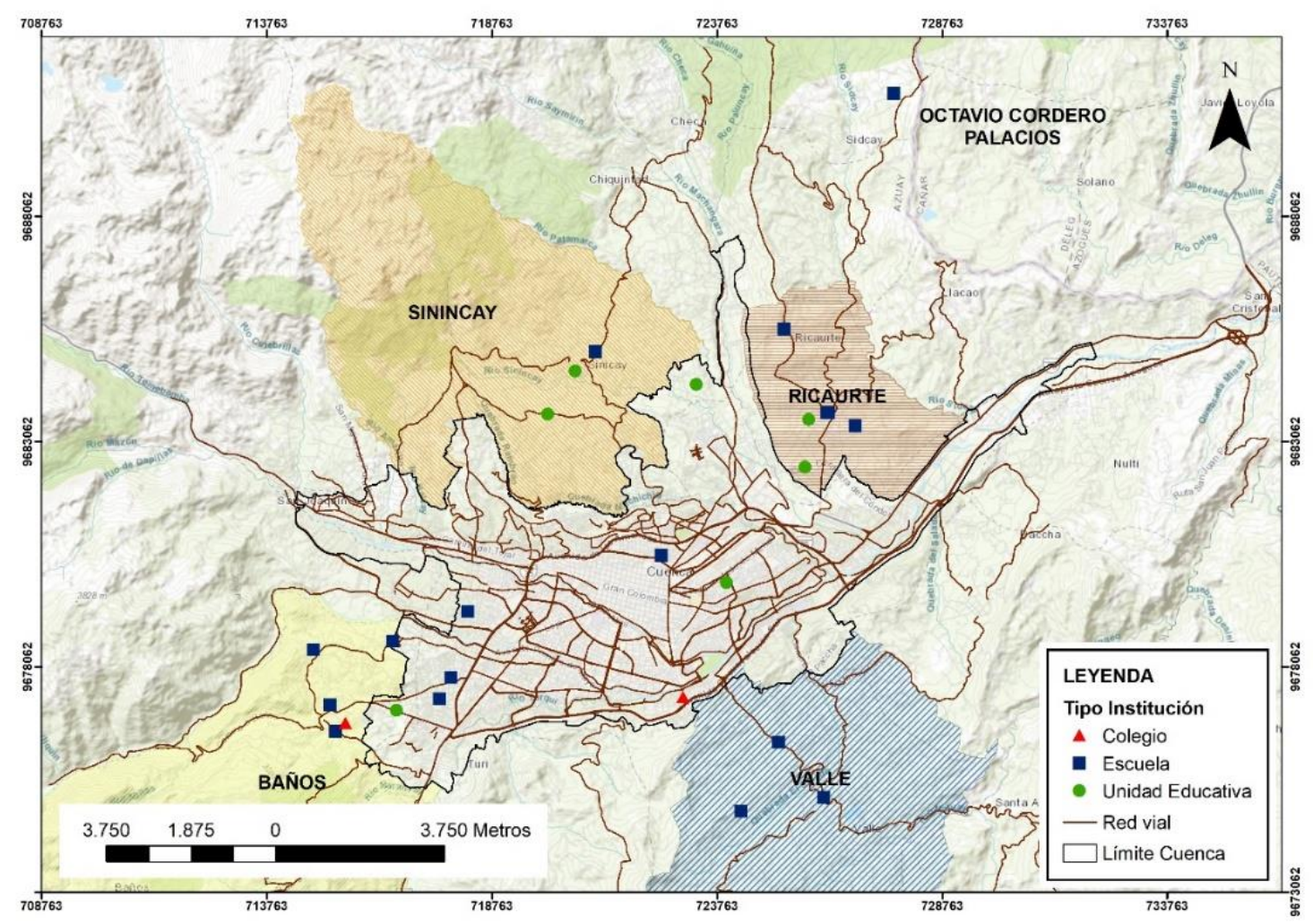

Figura 3: Distribución de centros educativos.

Las parroquias Sinincay y El Valle presentan datos representativos, Sinincay muestra un gran porcentaje de estudiantes que se movilizan dentro de la parroquia (94.7\%) y El Valle 
la de mayor porcentaje de traslado hacia la zona urbana (76.5\%). En el caso de las parroquias de Baños y Ricaurte, el mayor porcentaje se concentra en una movilidad interna (80.28 y $69.12 \%$ ) (Tabla 1).

Tabla 1: Porcentaje de traslado de estudiantes a centros educativos dentro y fuera de la parroquia de origen.

\begin{tabular}{lcc}
\multicolumn{1}{c}{ Parroquia } & \multicolumn{2}{c}{ Traslado } \\
\hline Baños & En la parroquia \% & Hacia la zona urbana \% \\
Ricaurte & 80.28 & 19.72 \\
Sinincay & 69.12 & 30.88 \\
El Valle & 94.69 & 5.31 \\
\hline
\end{tabular}

Con respecto a los modos de transporte se observa que los mayores porcentajes de estudiantes se movilizan a pie (54.38 \%), lo que sugiere que residen cerca de los centros educativos. Cabe mencionar que los estudiantes de las parroquias de Ricaurte y Baños son las que más realizan este tipo de desplazamientos cortos. El transporte público es el segundo medio utilizado (41.40\%), en todas las parroquias excepto en Baños, aquí el segundo medio es el vehículo propio, $16.9 \%$. Además, se evidencia que los estudiantes de las parroquias de El Valle y Sinincay son más dependiente del transporte público. El uso de otros modos de transporte como los vehículos de alquiler, vehículo propio, moto o bicicleta es relativamente bajo (2.94 \%) (Tabla 2).

Tabla 2: Análisis de medios de transporte por parroquia.

\begin{tabular}{|c|c|c|c|c|c|}
\hline & & & & & \\
\hline & \multirow{2}{*}{ Medio de transporte } & \multicolumn{4}{|c|}{ Parroquia rural } \\
\hline & & Baños & Ricaurte & Sinincay & El Valle \\
\hline \multirow{6}{*}{$\begin{array}{l}\text { Estudiantes por medio de } \\
\text { transporte }(\%)\end{array}$} & Transporte Colectivo & 15.5 & 20.4 & 49.4 & 54.4 \\
\hline & Vehículo de Alquiler & 5.6 & 2.7 & 1.2 & 2.9 \\
\hline & Vehículo Propio & 16.9 & 8.0 & 1.2 & 2.9 \\
\hline & Moto & 0.7 & - & - & - \\
\hline & Bicicleta & 0.7 & - & - & - \\
\hline & A Pie & 60.6 & 69.0 & 48.2 & 39.7 \\
\hline \multirow{6}{*}{$\begin{array}{l}\text { Tiempo promedio recorrido por } \\
\text { medio de transporte (minutos) }\end{array}$} & Transporte Colectivo & 26 & 33 & 28 & 34 \\
\hline & Vehículo de Alquiler & 29 & 17 & 10 & 12 \\
\hline & Vehículo Propio & 16 & 12 & 15 & 22 \\
\hline & Moto & 15 & - & - & - \\
\hline & Bicicleta & 10 & - & - & - \\
\hline & A Pie & 19 & 13 & 13 & 9 \\
\hline \multirow{6}{*}{$\begin{array}{l}\text { Distancia en kilómetros por } \\
\text { modo de transporte }\end{array}$} & Transporte Colectivo & 32.3 & 55.1 & 113.6 & 91.9 \\
\hline & Vehículo de Alquiler & 13.9 & 5.2 & 0.6 & 2.9 \\
\hline & Vehículo Propio & 30.3 & 13.7 & 1.0 & 7.1 \\
\hline & Moto & 0.9 & - & - & - \\
\hline & Bicicleta & 0.4 & - & - & - \\
\hline & A Pie & 63.2 & 42.8 & 39.6 & 11.7 \\
\hline
\end{tabular}

La tabla 2 muestra los tiempos promedio de desplazamiento de los estudiantes de las áreas de estudios en función de los medios utilizados. Se identifica que el mayor tiempo corresponde a la accesibilidad en transporte público colectivo, 30 minutos. Por otra parte, se observa que en la movilidad en vehículo propio los tiempos de recorrido son menores, y 
por tanto presentan mejores condiciones de accesibilidad, 16.25 minutos. Los desplazamientos a pie tienen un promedio de 13.5 minutos para los hogares que se ubican más próximos a los centros educativos.

Analizando las distancias de desplazamientos se observa que, existe un mayor recorrido de los estudiantes que utilizan transporte público colectivo $(73.23 \mathrm{~km})$, mostrando concordancia con respecto a los datos anteriormente analizados. En lo que respecta a la distancia recorrida a pie, también se refleja un dato significativo, en promedio $39.33 \mathrm{~km}$ (Tabla 2). En la parroquia Sinincay se recorre en promedio una mayor distancia (1.31 a 2.07 $\mathrm{km}$ ), principalmente por los estudiantes de 12 a 20 años. Esto puede representar que las condiciones de accesibilidad son más complejas, que no se cuenta con la infraestructura o equipamiento adecuado que permita mejor la accesibilidad, y que, a su vez, el sistema educativo no cuenta con la infraestructura suficiente (déficit de instituciones educativas en la parroquia), siendo además un elemento crítico su ubicación con respecto al requerimiento de estudiantes. En lo que respecta a la parroquia El Valle, se encontró que, en el rango de edad de 3 a 5 años, preescolar, se recorre en promedio una menor distancia $(0.26 \mathrm{~km})$, lo que puede considerarse como un aspecto factible dentro del análisis de la accesibilidad. En general, los estudiantes de 12 a 20 años es el que en promedio más distancia recorre (entre $0.91 \mathrm{~km}$ y $2.07 \mathrm{~km}$ ) (Tabla 3).

Tabla 3: Distancia promedio recorrida por grupo etario expresada en $\mathrm{km}$.

\begin{tabular}{lccc} 
Parroquia & $3-5$ años & $6-11$ años & 12-20 años \\
\hline Baños & 1.06 & 0.96 & 0.91 \\
Ricaurte & 0.99 & 0.71 & 1.25 \\
Sinincay & 1.31 & 1.32 & 2.07 \\
El Valle & 0.26 & 1.09 & 1.68
\end{tabular}

La tabla 4 muestra un alto promedio de distancia recorrida a través del transporte público colectivo, entre $1.47 \mathrm{~km}$ y $2.48 \mathrm{~km}$. Esto puede representar una mayor factibilidad para acceder a este modo de transporte, en comparación con los otros valores estudiados. Sin embargo, la accesibilidad a pie también indica valores significativos $(0.3 \mathrm{~km}$ a $4.5 \mathrm{~km})$, comprendiendo a este como un dato esencial para visibilizar la importancia de este modo de movilidad dentro de las parroquias. Una consideración importante es que, al analizar los valores de promedio de distancia, de los tres grupos etarios, se muestra una diferencia significativa en lo que respecta a su recorrido, lo que permite establecer un indicador con respecto a la movilidad de estudiantes que asisten a un nivel básico de educación (escuela), quienes recorren menos distancia, sobre estudiantes de niveles superiores (colegios e institutos superiores tecnológicos), que recorren una distancia de mayor extensión en todas las parroquias en estudio. 
Tabla 4: Distancia promedio recorrida por medio de transporte.

\begin{tabular}{lccccccc} 
Parroquia & $\begin{array}{c}\text { Grupo etario } \\
(\mathrm{años})\end{array}$ & $\begin{array}{c}\text { Transporte } \\
\text { Colectivo } \\
(\mathrm{km})\end{array}$ & $\begin{array}{c}\text { Vehículo de } \\
\text { Alquiler }(\mathrm{km})\end{array}$ & $\begin{array}{c}\text { Vehículo } \\
\text { Propio } \\
(\mathrm{km})\end{array}$ & $\begin{array}{c}\text { Moto } \\
(\mathrm{km})\end{array}$ & $\begin{array}{c}\text { Bicicleta } \\
(\mathrm{km})\end{array}$ & $\begin{array}{c}\text { A Pie } \\
(\mathrm{km})\end{array}$ \\
\multirow{5}{*}{ Baños } & $3-5$ & 1.48 & 2.28 & 1.15 & - & - & 0.69 \\
& $6-11$ & 1.32 & 1.52 & 1.33 & - & - & 0.77 \\
\multirow{5}{*}{ Ricaurte } & $12-20$ & 1.50 & 1.00 & 1.48 & 0.86 & 0.43 & 0.66 \\
& $3-5$ & 1.91 & - & - & - & - & 0.74 \\
& $6-11$ & 1.59 & 1.55 & 1.21 & - & - & 0.51 \\
\multirow{3}{*}{ Sinincay } & $12-20$ & 2.85 & 2.08 & 3.02 & - & - & 0.53 \\
& $3-5$ & 1.79 & - & 0.99 & - & - & 0.73 \\
& $6-11$ & 2.10 & - & - & - & - & 0.78 \\
El Valle & $12-20$ & 2.95 & 0.59 & - & - & - & 1.03 \\
& $3-5$ & - & - & - & - & - & 0.74 \\
& $6-11$ & 4.19 & 1.05 & - & - & - & 0.33 \\
& $12-20$ & 2.40 & 1.84 & 3.53 & - & - & 0.67
\end{tabular}

\subsection{Accesibilidad a áreas consolidadas}

Para identificar las áreas consolidadas se determinó el incremento de medidores de energía eléctrica (cada punto representa espacialmente una vivienda), en el período comprendido entre el 2010 - 2020, se identificó un incremento total de 14173 medidores. De acuerdo con el modelo de densidades ponderadas "Kernel" (Guzmán, 2020) se delimitó las zonas que en los últimos años se han densificado. El modelo arrojó como resultados una desviación estándar de 59.32 medidores $/ \mathrm{km}^{2}$, un promedio de 18.37 medidores $/ \mathrm{km}^{2}$ que a partir de los 300 medidores $/ \mathrm{km}^{2}$ la dispersión en los valores de densidad disminuye considerablemente. Por lo cual se estableció que las zonas con densidades superiores a 300 medidores $/ \mathrm{km}^{2}$ se encuentran en proceso de consolidación urbana (Figura 4).

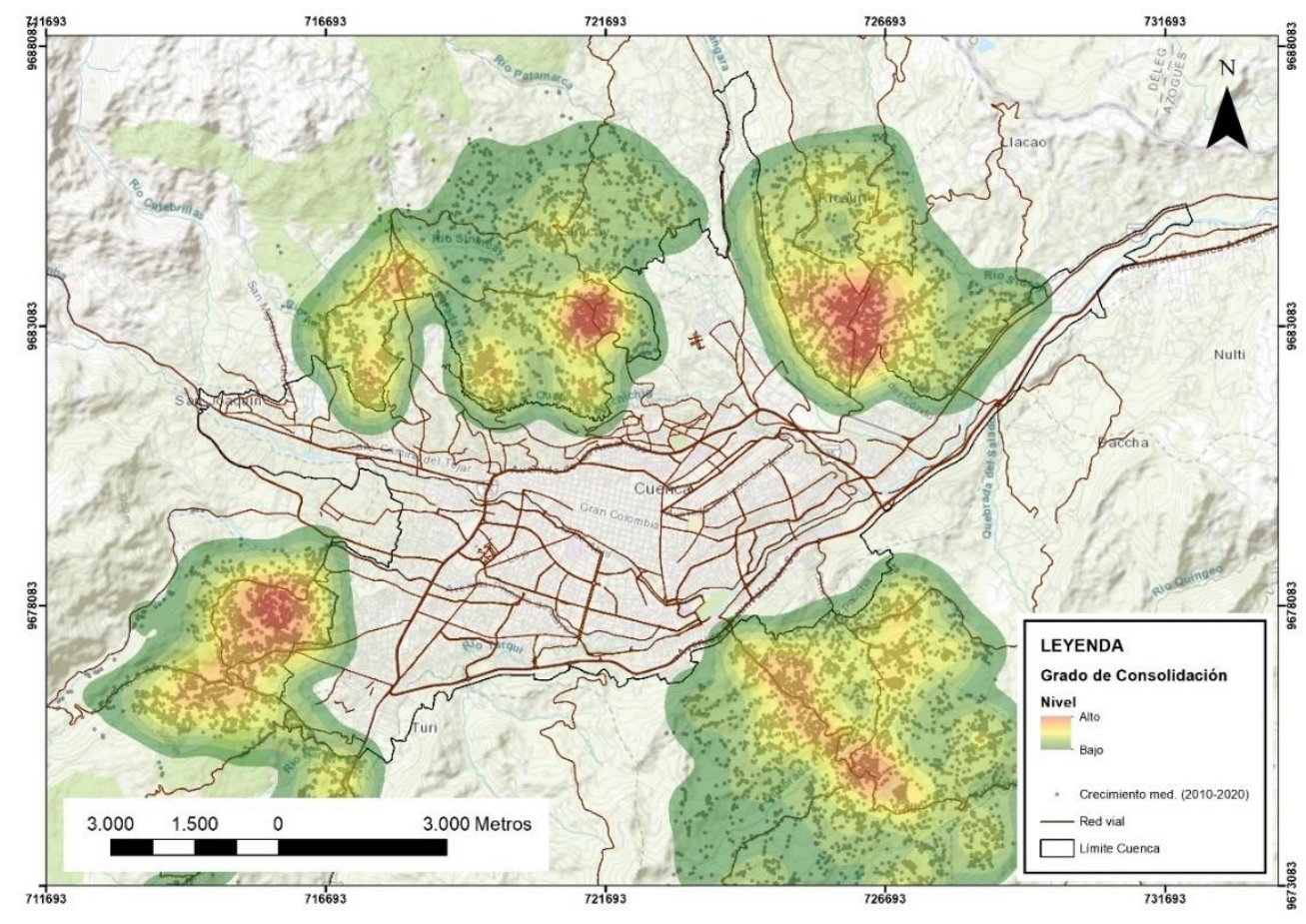

Figura 4: Análisis de densidad y consolidación de áreas urbanas. 
El cálculo del Índice de Moran a través de SIG establecerá la existencia de una relación basada en la ubicación de medidores de energía eléctrica asumiendo a cada uno de estos como una unidad de vivienda. La puntuación " $\mathrm{z}$ " corresponde a la desviación estándar y el valor " $\mathrm{P}$ " está asociado a la probabilidad de una curva ajustada a una distribución conocida para el área de estudio. La figura 5 muestra una correlación de 2.58 entre el incremento de densidad de medidores de energía eléctrica al año 2020 con respecto a datos del año 2010. Es decir, el crecimiento se ha desarrollado de manera céntrica con respecto a la población existente, sin existir picos relacionados a distancias largas o fuera del ámbito de su ubicación.

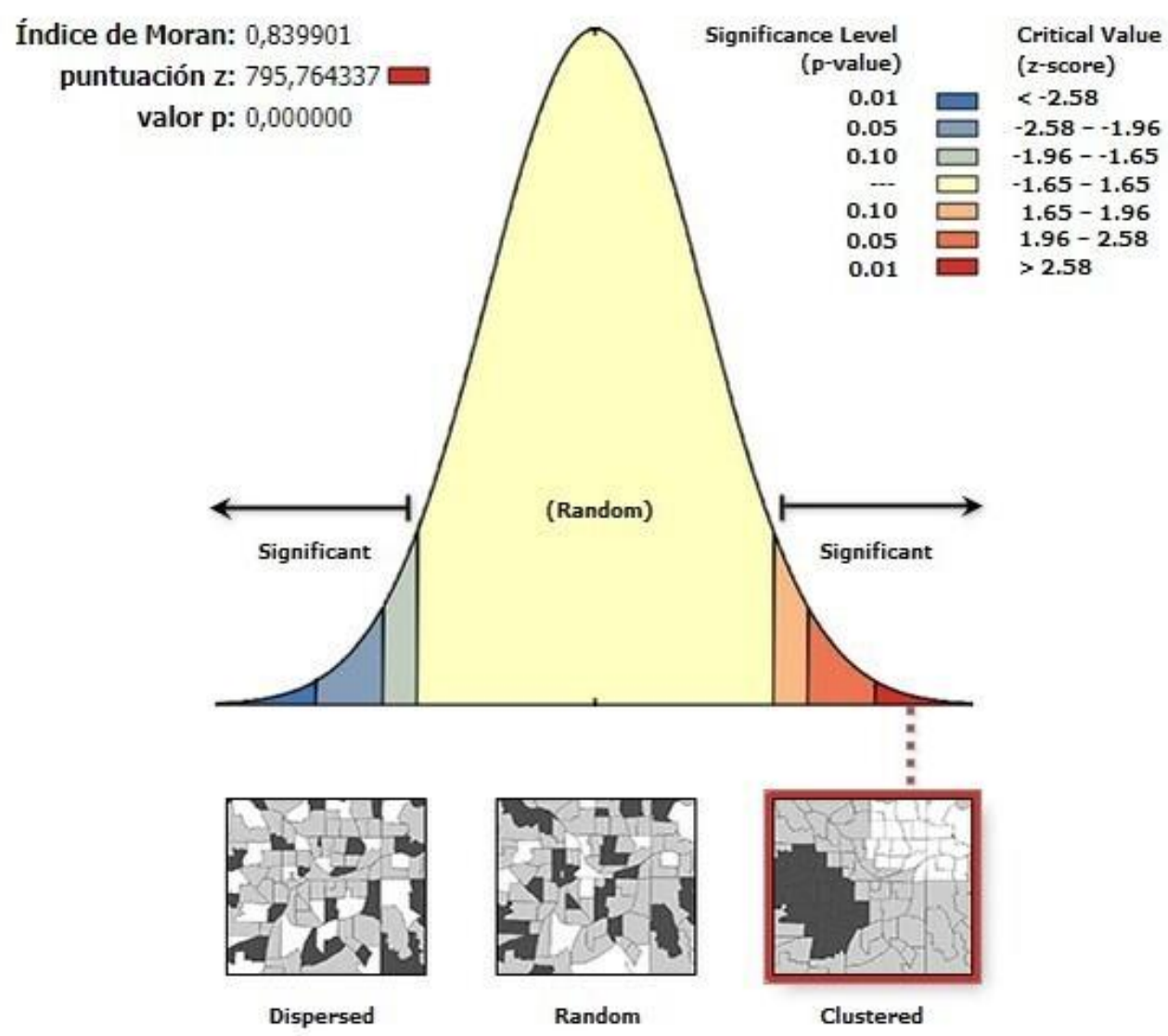

Figura 5: Cálculo del índice de Moran para determinar autocorrelación con base en ESRI (2022).

Dado el puntaje $\mathrm{z}$ de 795764, hay menos del $1 \%$ de probabilidad de que este patrón agrupado pueda ser un resultado aleatorio, es decir, asegura la presencia de una autocorrelación en los datos analizados.

En la figura 6 se muestra el cálculo del Índice G de Getis Ord a través del cual se determina una alta correlación entre el crecimiento poblacional y la ocupación del suelo. En este sentido, los datos de crecimiento poblacional del año 2020 presentan una estrecha relación con los datos existentes desde el año 2010, representado en una mayor ocupación del suelo con infraestructura, tanto habitacional como comercial, así como el incremento del número de pisos de las edificaciones. Finalmente, el gráfico presenta que existe menos del 1\% de probabilidad de que este patrón de agrupación sea aleatorio. 


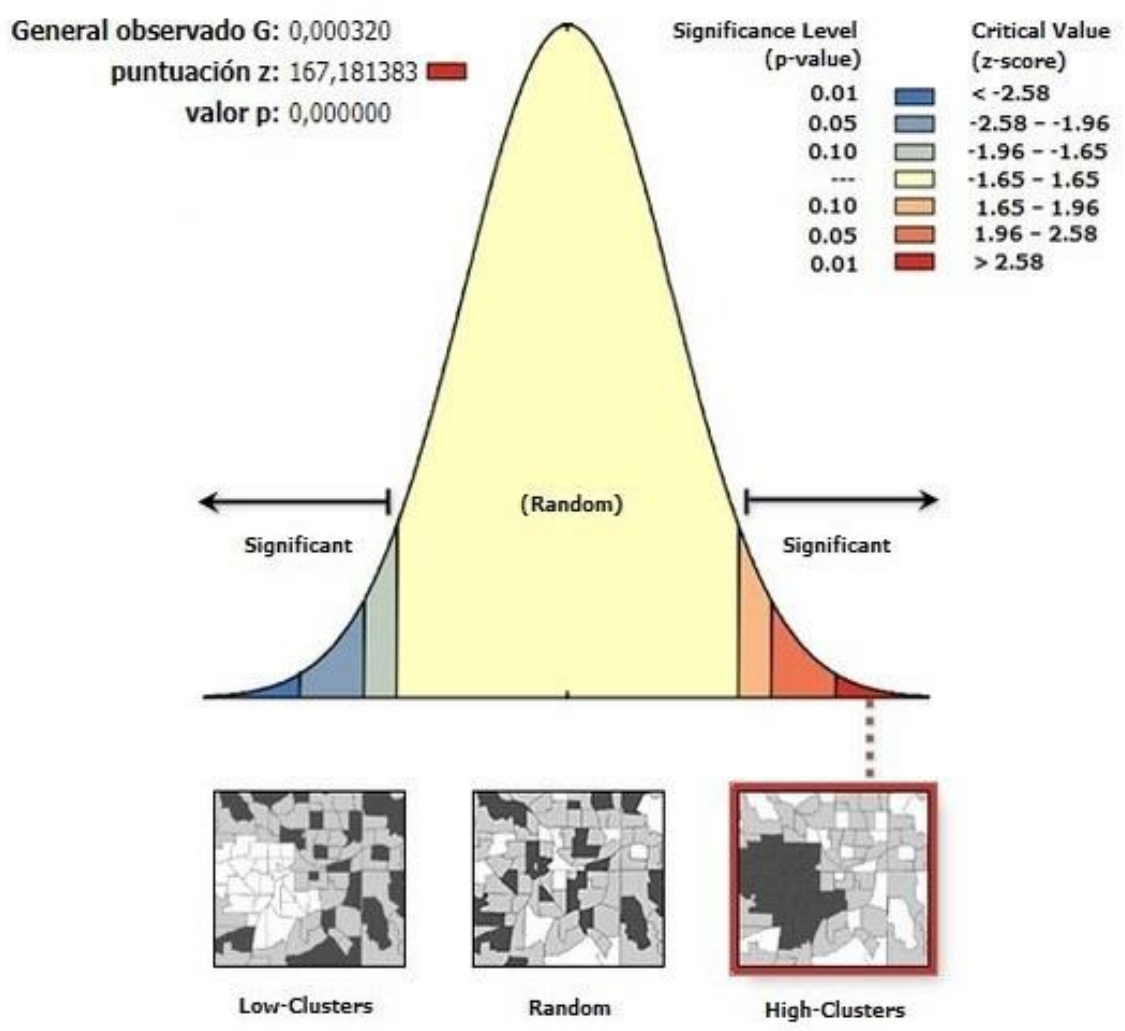

Figura 6: Cálculo del Índice G de Getis Ord para el grado de autocorrelación con base en ESRI (2022).

Una vez determinadas las zonas con mayor consolidación se analiza los medidores localizados en estas áreas y su relación tiempo - distancia con los centros de educación identificados en el análisis de hogar, mediante líneas de deseo (líneas imaginarias que pasan sobre el trayecto más eficiente o utilizado por un peatón o ciclista). Estas líneas precisan las relaciones descritas anteriormente. Para una mayor precisión del modelo se utilizaron 2779 puntos de medidores localizados espacialmente en las zonas evaluadas como consolidadas. La figura 7 ilustra cómo se generan varias líneas (13000) de un punto a otro trazando distancias que van desde los $5 \mathrm{~m}$ hasta $\operatorname{los} 10000 \mathrm{~m}$.

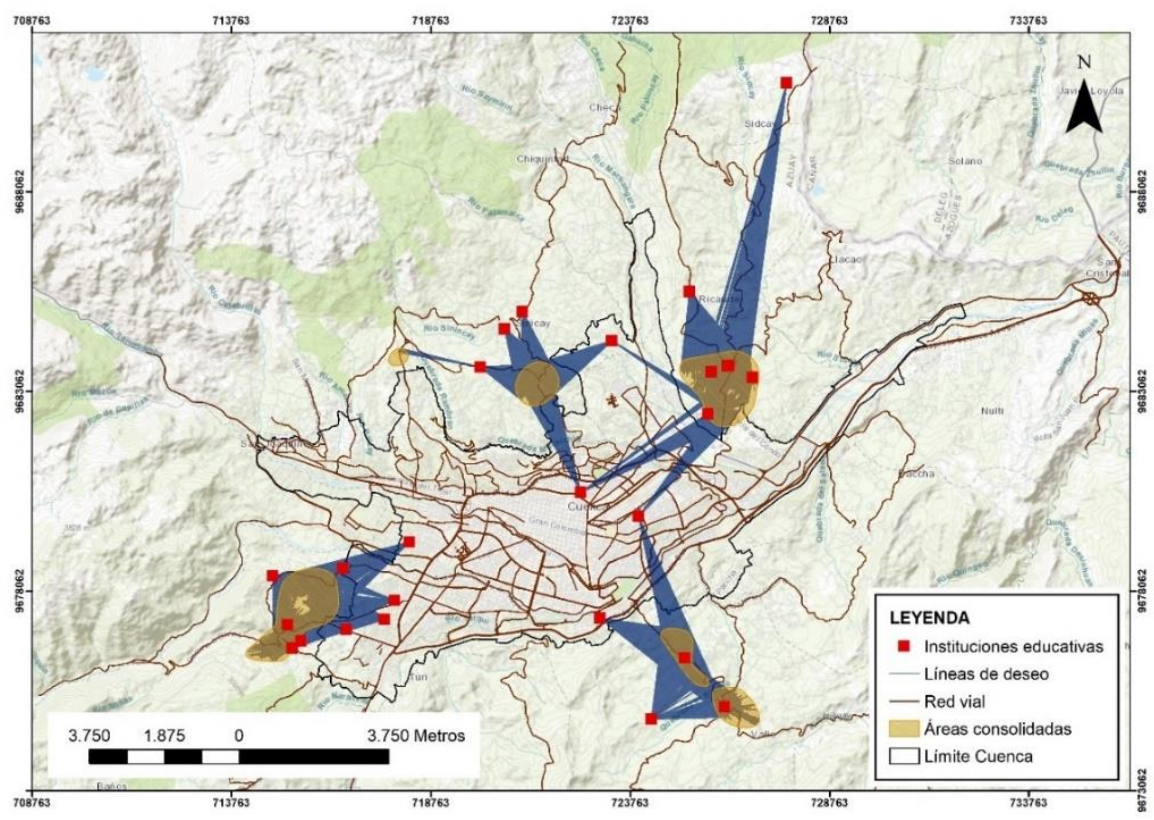

Figura 7: Análisis de líneas más probables origen - destino. 
El modelo espacial de la figura 8 determina que, a los estudiantes les toma entre 1 a 60 minutos es decir distancias menores a 100 m hasta $9 \mathrm{~km}$ aproximadamente, al centro educativo más cercano dependiendo de su ubicación de origen (vivienda) además precisa que en ciertas áreas consolidadas los desplazamientos no toman más de 20 minutos. Sin considerar variables como la topografía del terreno, condición física, entre otros.

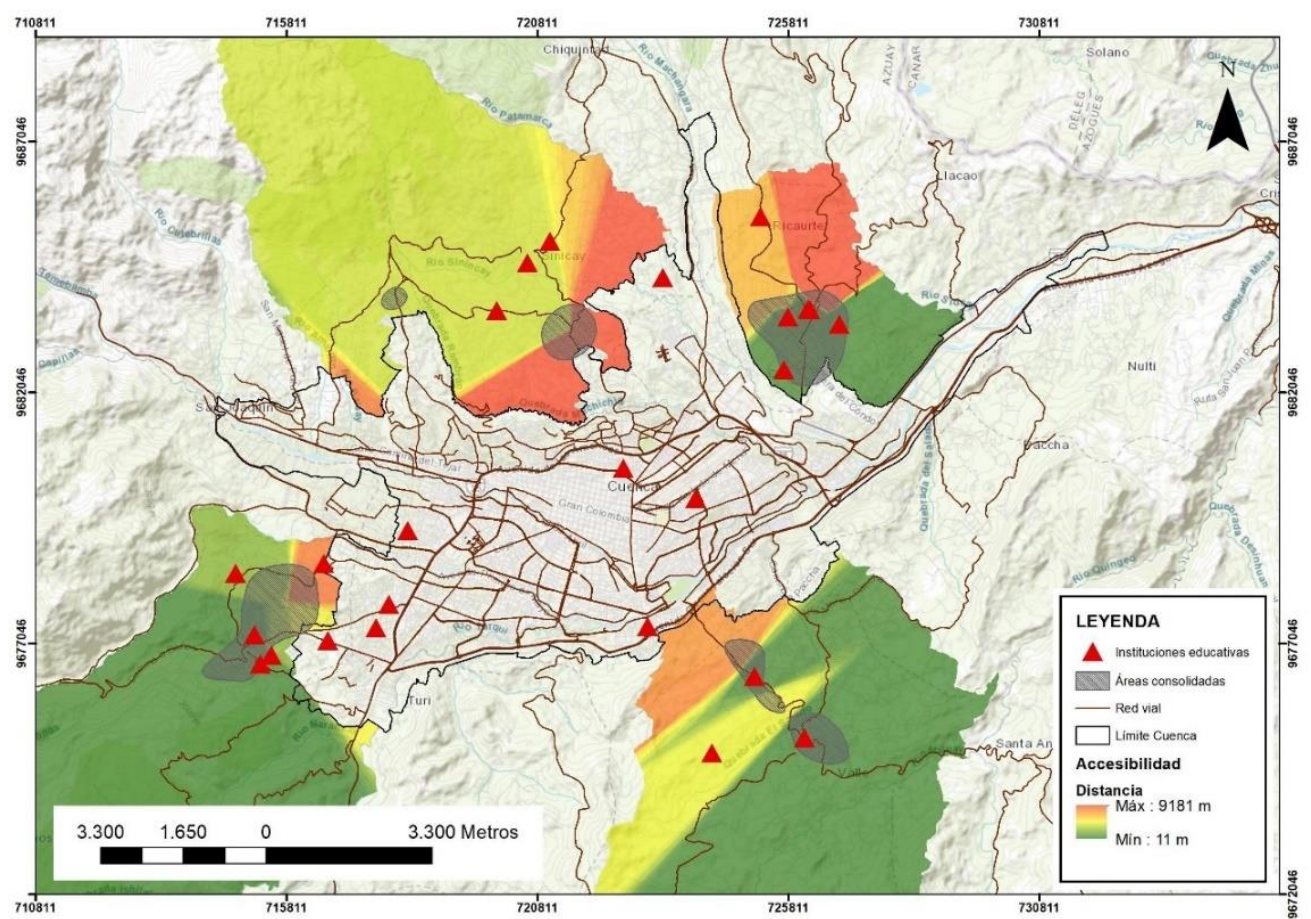

(a) Accesibilidad por distancia a los centros educativos.

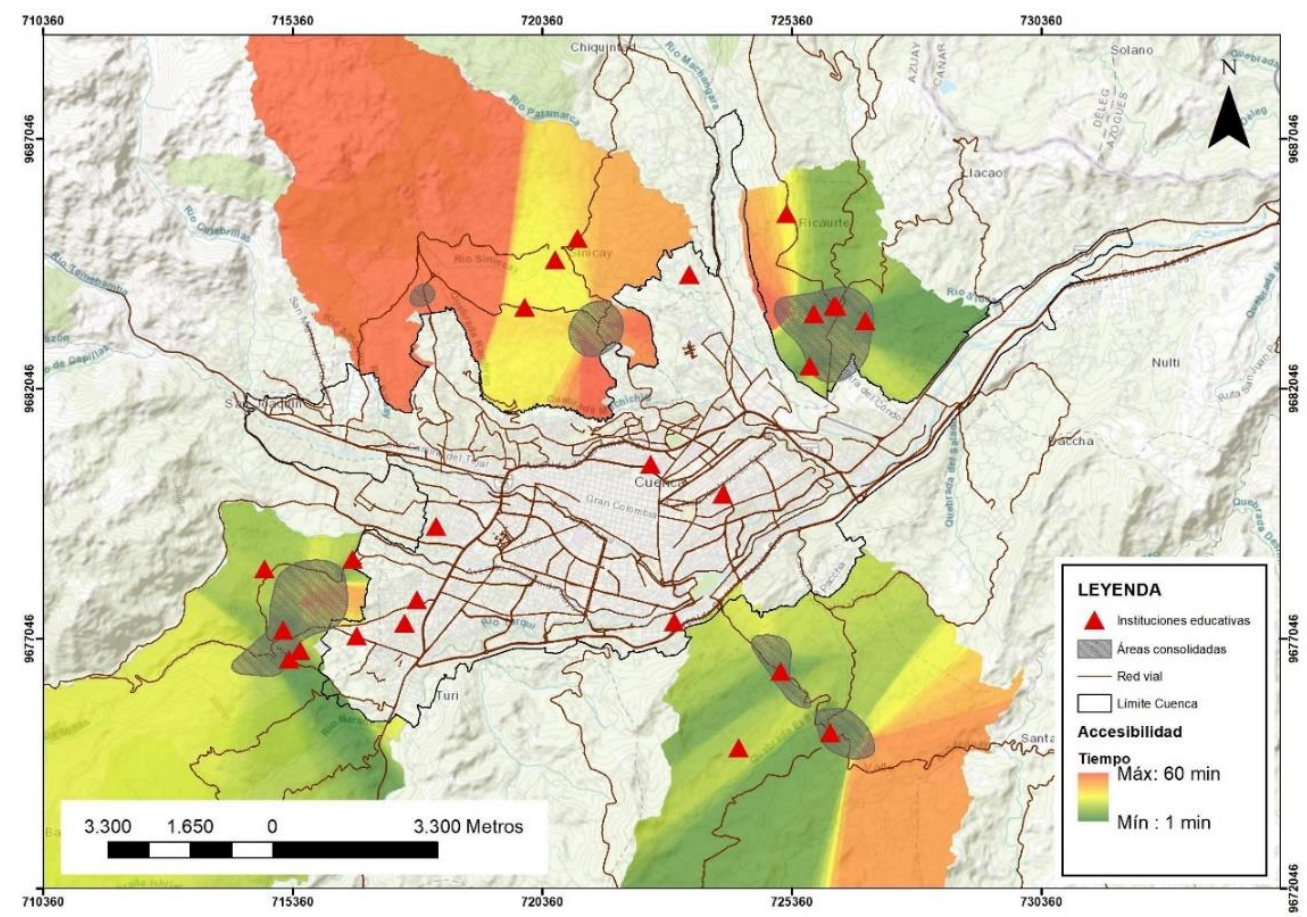

(b) Accesibilidad por tiempo a los centros educativos.

Figura 8: Análisis de accesibilidad por distancia y tiempo a los centros educativos. (a) Accesibilidad por distancia a los centros educativos. (b) Accesibilidad por tiempo a los centros educativos. 
Finalmente, tras el análisis de los radios de influencia de los centros educativos de acuerdo a tres rangos de estudio propuestos por De la Fuente et al. (2013), en la tabla 5 se muestra que el $65 \%$ de la población estimada cuenta con una accesibilidad baja, el $27 \%$ tiene una accesibilidad media y apenas el $8 \%$ tienen una accesibilidad alta, en función de los rangos establecidos.

\begin{tabular}{|c|c|}
\hline RANGO & $\begin{array}{l}\text { ACCESIBILIDAD } \\
\text { Valores Relativos }\end{array}$ \\
\hline Alta $(0-500 m)$ & $8 \%$ \\
\hline Media (501-1000m) & $27 \%$ \\
\hline Baja $(1000 m)$ & $65 \%$ \\
\hline
\end{tabular}

\section{Discusión}

La aplicación de la metodología propuesta por esta investigación analiza la accesibilidad a los centros educativos en el área periurbana de Cuenca en Ecuador. En una primera instancia, a través del uso de datos sobre las características de movilidad de estudiantes, se evidencia que los integrantes del grupo etario de 12 a 20 años correspondiente al nivel secundario, son los que más se desplazan. Por otro lado, en niveles de educación preescolar/primaria se observa menores desplazamientos, lo que confirmaría la tendencia indicada con respecto a la concentración del nivel de estudio en las parroquias de Baños y Ricaurte.

Al igual que lo reportado por De la Fuente et al. (2013) esta investigación plantea un análisis de la accesibilidad en función de tres rangos: < 500 m, entre 500 y 1000 m y > 1000 m, este procedimiento permite determinar zonas que posteriormente pueden ser útiles para la definición de intervenciones, planes especiales e incluso políticas locales. Este tipo de análisis son fundamentales en ciudades de América Latina, dado el rápido crecimiento de su población como lo señala Châu (2003).

En Ecuador no existen parámetros de accesibilidad a centros educativos establecidos a nivel nacional, ha quedado en manos de los gobiernos autónomos municipales el establecer parámetros que les permita gestionar la reserva de suelo, puesto que la dotación de equipamientos educativos es gestión del gobierno central. Sin embargo, no todos los cantones cuentan con parámetros que les permita medir los niveles de accesibilidad existente hacia los centros de educación. Estos parámetros en conjunto con información sobre el tamaño de la población, como señala Jega et al. (2012), pueden servir para la dotación del servicio de transporte público y la configuración de nuevas rutas o la ampliación de las existentes.

Por otro lado, en lo que respecta a las edades de educación preescolar y primaria, esta se caracterizó por una accesibilidad a las instituciones educativas a pie, es decir, sin la necesidad de ningún vehículo automotor o bicicleta, dinámicas similares a lo establecido por Annessi et al. (2018). Lo que sugiere que se prioriza la accesibilidad a pie al centro de 
educación primaria a través de la planificación de escuelas relativamente cercanas a las áreas pobladas.

El medio de transporte público colectivo cuenta también con un alto porcentaje de ocupación sobre todo en estudiantes de nivel secundario, su uso obedece a la necesidad de alcanzar el destino final en menor tiempo. Conforme lo indicado por la Organización de las Naciones Unidas para la Educación, la Ciencia y la Cultura (UNESCO, 2013) la falta de equidad sigue afectando a la población de las zonas rurales, quienes no pueden costear este servicio y deben recorrer distancias más largas; por lo que promover estrategias de movilidad a bajo o nulo costo aseguraría que los estudiantes de medios rurales puedan ejercer en igualdad de condiciones su derecho a la educación (Raikes et al., 2015), o a su vez aplicar medidas de integración entre el transporte regular y escolar (Badía, 2016).

Los altos porcentajes de estudiantes que se movilizan en determinados medios de transporte pueden reflejar que se ha desarrollado un equipamiento e infraestructura apropiada, tanto para la movilidad motorizada, así como para la no motorizada. Los modos de transporte analizados reflejan un alto porcentaje de traslado a pie, seguido por el uso del transporte público colectivo, lo que refleja un equipamiento público adecuado que permite que estos modos se desarrollen de mejor manera. Sin embargo, los estudiantes que utilizan transporte colectivo se trasladan en promedio una mayor distancia, y requieren de mayor tiempo para acceder a las instituciones educativas (26 a 33 minutos). Esto indica que, aunque muchos de los estudiantes se trasladan a pie, los restantes requieren más tiempo y una mayor distancia de traslado hacia su destino.

Elementos esenciales como el tiempo usado para el traslado hacia las instituciones educativas representa un aspecto importante en la accesibilidad. Se ha determinado que en lo que respecta al transporte colectivo, en promedio se requiere de entre 16 y 34 minutos de recorrido, y con respecto al traslado a pie, un promedio de entre 9 y 19 minutos. Estos datos de accesibilidad a pie concuerdan con lo establecido por Alvarez et al. (2019) que en su estudio de accesibilidad peatonal a servicios educativos asume como regular tiempos entre 10 a 20 minutos. Siendo una accesibilidad óptima destinos alcanzados a pie en menos de 10 minutos.

La distancia promedio recorrida mediante el uso del transporte público colectivo representa la mayor proporción con respecto a los otros modos, con un promedio entre $1.47 \mathrm{~km}$ y 2.7 $\mathrm{km}$, y se centra de manera más intensiva en los rangos de edad de niveles secundario o técnico. Esto define un claro indicador sobre la falta de infraestructura educativa de dicho nivel en la zona de estudio. Incluso si cuentan con un bajo porcentaje de movilidad de estudiantes secundarios, es imperativo contar con la infraestructura y equipamiento educativo adecuado. Comparando los datos obtenidos de distancias promedio recorridas por los estudiantes en el periurbano de Cuenca en Ecuador, con los resultados publicados por Annessi et al. (2018) para las localidades de Maipú y General Guido, muestran que las distancias hacia los centros educativos en este estudio son cortas.

De acuerdo con el segundo enfoque que toma la presente investigación respecto a la accesibilidad y su relación a las áreas consolidadas, se definieron ámbitos de estudio más precisos vinculados a i) sus características y dinámicas territoriales; y ii) su ubicación 
geográfica cuyas pendientes son bajas cerca de vías arteriales. Dichos ámbitos fueron definidos a través de herramientas SIG, puesto que ofrecen métodos innovadores para contribuir a los procesos de planificación de infraestructura educativa como señala Köse et al. (2021) y Pino et al. (2019). De este análisis se estableció que la zona consolidada de Baños al poseer el mayor porcentaje de centros educativos, la población requiere movilizarse distancias más cortas a comparación de los estudiantes localizados en Ricaurte, que si bien cuentan con varias unidades educativas a su alcance de acuerdo con el modelo espacial alineado a las encuestas se reflejan varios movimientos hacia la ciudad de Cuenca e incluso hacia otras parroquias.

\section{Conclusiones}

De acuerdo con la relación de tiempo y distancia vinculados a los desplazamientos se determinó que existe una baja oferta de infraestructura educativa en las zonas consolidadas de El Valle y Sinincay, mientras que en el caso de la parroquia de Baños los tiempos de desplazamiento son más extensos; debido a que, a pesar de tener centros de educación cercanos los estudiantes se trasladan a la ciudad de Cuenca sea por motivos de calidad de enseñanza o porque cuentan con vehículo propio. Además, se estima que, si existiese un incremento y consolidación de la población con el trascurso del tiempo, estos equipamientos no serán suficientes para la población que se desplaza localmente, aumentando proporcionalmente los viajes hacia la ciudad de Cuenca y ocasionalmente colapsar el sistema público de transporte en las áreas periurbanas.

Analizando los modelos espaciales se determinó que existe población en edad estudiantil que, a pesar de contar con una cercanía espacial a centros de educación en las zonas ya consolidadas de la parroquia de Ricaurte y Baños, por la falta aparente de conectividad relacionada a la infraestructura vial existente, los estudiantes ocupan mayores tiempos de desplazamiento recorriendo proporcionalmente una mayor distancia por vías alternas. Lo que indicaría que mejorar el sistema vial e incluso proponer espacios o corredores por los cuales los estudiantes se puedan desplazar a pie o en bicicleta mejoraría su movilidad y por ende su accesibilidad.

Los resultados de este estudio sugieren que es de vital importancia generar políticas públicas vinculadas a un adecuado ordenamiento territorial que promueva los principios de equidad, eficiencia y justicia espacial en lo que respecta a accesibilidad a equipamientos de carácter colectivo. Actualmente los SIG constituyen una herramienta de gran importancia vinculada a procesos de la gestión del territorio. Su propósito es el de analizar áreas que presentan serias dificultades de accesibilidad, para que se generen estudios que faciliten la ejecución de acciones que eviten el abandono de estos espacios y posteriormente provoquen deserción escolar. Una infraestructura apropiada para el sistema de educación pública garantiza una prospectiva de equidad, tanto social como territorial.

Bajo este contexto, es necesario analizar nuevas líneas de investigación que comprueben, desde un enfoque socioeconómico, si las instalaciones educativas correspondientes al área de estudio se encuentran subutilizadas o sobre utilizadas según el caso. Además, es necesario nuevos enfoques con indicadores de calidad de educación, oferta y demanda. Esto 
con el fin de determinar si estas variables definen aún más las posibles brechas que pueden existir entre espacios urbanos y rurales.

\section{Conflicto de Interés}

Los autores declaramos que en la presente investigación no existen conflictos de interés de naturaleza alguna.

\section{Fuente de financiamiento}

Dirección de Investigación de la Universidad de Cuenca.

\section{Agradecimiento}

Este estudio se realizó en el marco del proyecto «Estudio de la relación entre el coste del suelo y la vulnerabilidad. Efectos sobre los patrones de ocupación, proyecto de investigación del Departamento de Investigación de la Universidad de Cuenca (DIUC) a quien dirigimos nuestro agradecimiento.

\section{Contribución de los autores}

En concordancia con la taxonomía establecida internacionalmente para la asignación de créditos a autores de artículos científicos (https://casrai.org/credit). Los autores declaran sus contribuciones en la siguiente matriz:

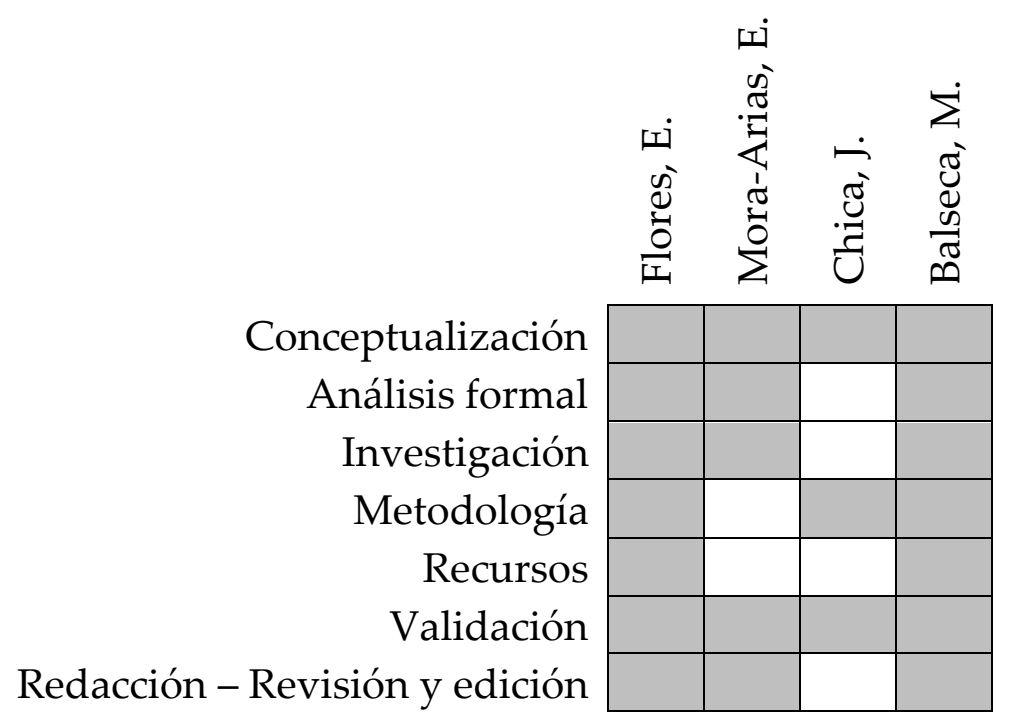

\section{Referencias}

Ministerio de Educación. (2012). Acuerdo 0483-12: Por el cual se establecen las normas técnicas y estándares de infraestructura educativa. Recuperado de: https://educacion.gob.ec/wp-content/uploads/downloads/2013/01/ACUERDO-48312.pdf 
Alvarez, M., Quirós, E., \& Gutiérrez, J. (2019). Accesibilidad peatonal a los servicios educativos de la ciudad de Ibagué-Tolima, Colombia. Revista Cartográfica, (98), 305328. https://doi.org/10.35424/RCARTO.I98.152

Annessi, G. J., Bachex, V., \& Demirta, P. (2018). La oferta de la educación en los espacios rurales como factor de retención demográfica. El caso de la región del este de la provincia de Buenos Aires. En I Congreso Iberoamericano de Docentes. Algeciras, Cádiz, España. Recuperado de: http://formacionib.org/congreso/257.pdf

ESRI (2022). Cómo funciona autocorrelación espacial (I de Moran global). ArcMap de https://desktop.arcgis.com/es/arcmap/latest/tools/spatial-statistics-toolbox/h-howspatial-autocorrelation-moran-s-i-spatial-st.htm

Badía, L. (2016). Las posibilidades de integración del transporte escolar y regular en Aragón. Geographicalia, 68, 1-24. https://doi.org/10.26754/ojs_geoph/geoph.2016681579

Chaparro, I., \& Hernández, V. (2020). La reconfiguración de los subcentros de empleo en Ciudad Juárez, Chihuahua, 2004-2014. Región y Sociedad, 32, e1268-e1268. https://doi.org/10.22198/RYS2020/32/1268

Châu, T. N. (2003). Demographic aspects of educational planning. UNESCO: International Institute for Educational Planning. Recuperado de: http://www.iiep.unesco.org/en/publication/demographic-aspects-educationalplanning

De la Fuente, H., Rojas, C., \& Salado, M. (2013). Distribución de los equipamientos educativos. Evidencias de inequidad espacial en la educación del área metropolitana de Concepción. GeoFocus. Revista Internacional de Ciencia y Tecnología de la Información Geográfica, (13-2), 231-257. Recuperado de https://www.geofocus.org/index.php/geofocus/article/view/296/144

De Oliveira, G., \& Hurtado, C. (2017). Expansión urbana y cohesión territorial en el proceso de construcción la ciudad metropolitana en Andalucía. Cuadernos Geográficos, 56(2), 223-244. https://doi.org/10.30827/CUADGEO.V56I2.5275

Escobar, D. A., Urazán, C. F., \& Moncada, C. A. (2017). Análisis de Cobertura Urbana de los Nodos de Actividad Primaria Mediante un Estudio de Accesibilidad Territorial en Quibdó (Colombia). Información Tecnológica, 28(5), 177-190. https://doi.org/10.4067/S0718-07642017000500018

GAD Municipal del Cantón Cuenca. (2015). Plan de Desarrollo y Ordenamiento Territorial del Cantón Cuenca Actualización 2015 (p. 750). Cuenca, Ecuador. Recuperado de https://multimedia.planificacion.gob.ec/PDOT/descargas.html

Gajardo, M. (2014). Educación y desarrollo rural en América Latina. Reinstalando un campo olvidado de las políticas educativas. Revista Iberoamericana de Evaluación Educativa, 7(3), 15-27. Recuperado de: https://revistas.uam.es/riee/article/view/3099

Guzmán, J. (2020). Localización óptima de viveros ornamentales con Sistemas de Información Geográfica (SIG) en la ciudad de Bogotá, Colombia. IDEA Construcción y Madera, 2(2), 78-87. http://revistas.sena.edu.co/index.php/idea/article/view/3057 
Hernandez, J., Martínez, B., Méndez, J., Pérez, R., Ramírez, J., \& Navarro, H. (2009). Rurales y periurbanos: una aproximación al proceso de conformación de la periferia poblana. Papeles de Población, 15, (61), 275-295. Recuperado de http://www.scielo.org.mx/scielo.php?pid=S140574252009000300011\&script=sci_abstract

Hidayati, I., Tan, W., \& Yamu, C. (2021). Conceptualizing Mobility Inequality: Mobility and Accessibility for the Marginalized. Journal of Planning Literature, 36(4), 496-507. https://doi.org/10.1177/08854122211012898

Instituto Nacional de Estadística y Censos (2010a). Nivel de instrucción educativa por parroquia del cantón Cuenca. https://www.ecuadorencifras.gob.ec/institucional/home/

Instituto Nacional de Estadística y Censos (2010b). Población por edad y por parroquia. https://www.ecuadorencifras.gob.ec/institucional/home/

Instituto Nacional de Estadística y Censos (2010c). Proyección cantonal total 2010 - 2020. https://www.ecuadorencifras.gob.ec/institucional/home/

Jega, I., Comber, A., \& Brunsdon, C. (2012). Population estimation in small areas: combining dasymetric mapping with pycnophylactic interpolation. In GISUK 2012 The Proceedings. At: Lancaster University. United Kingdom. Recuperado de: https://www.geos.ed.ac.uk/ gisteac/proceedingsonline/GISRUK2012/Papers/presen tation-56.pdf

Köse, M., Koçyiğit, M., Erdem, C., \& Jega, I. M. (2021). An evaluation of accessibility to preschool education institutions using geographic information systems. Education and Information Technologies, 26, 4307-4328. https://doi.org/10.1007/S10639-021-104827

Muñoz-Raskin, R. (2010). Walking accessibility to bus rapid transit: Does it affect property values? The case of Bogotá, Colombia. Transport Policy, 17(2), 72-84. https://doi.org/10.1016/J.TRANPOL.2009.11.002

Navarrete, M., \& López, A. M. (2019). Importancia de los espacios comunes: una adaptación de la técnica de interpolación espacial Invese Distance Weighted (IDW) en la predicción de datos socioeconómicos ausentes. En José Gasca Zamora y Serena Eréndira Serrano Oswald (Ed.), Regiones desplazamientos y geopolítica: agenda pública para el desarrollo territorial. Universidad Nacional Autónoma de México y Asociación Mexicana de Ciencias para el Desarrollo Regional A.C, Coeditores, Ciudad de México. Recuperado de http://ru.iiec.unam.mx/4646

Nieto, A., \& Márquez, N. (2018). Análisis de la distribución espacial de equipamientos educativos (0-16 años) en Extremadura a escala de detalle. Boletín de La Asociación de Geógrafos Españoles, (77), 493-520. https://doi.org/10.21138/bage.2549

Municipio del Distrito Metropolitano de Quito. (2003). Ordenanza 0095: Por la cual se establece el régimen del suelo del Distrito Metropolitano de Quito. 22 de agosto de 2003 (pp. 42-43). Recuperado de: http://www7.quito.gob.ec/mdmq_ordenanzas/Ordenanzas/ORDENANZAS\%20A\% C3\%91OS\%20ANTERIORES/ORDM-095\%20\%20NUEVO\%20REGIMEN\%20DEL\%20SUELO.pdf 
Pino, M., Astudillo, A., Aguirre, J., \& Salazar, A. (2019). Memoria social, cultura política y derecho a la ciudad. Un análisis en dos espacios públicos en Cuenca, Ecuador. Revista INVI, 34(96), 53-75. https://doi.org/10.4067/S0718-83582019000200053

Puchades, X. (2017). Estimaciones de densidad no paramétrica vía KERNEL (Tesis de Grado en Matemática Computacional). Castelló, España: Universitat Jaume I. Recuperado de: http://repositori.uji.es/xmlui/handle/10234/173690

Pueyo, Á. (1991). El sistema de información geográfica: un instrumento para la planificación y gestión urbana. Geographicalia, (28). 175-192. https://doi.org/10.26754/OJS_GEOPH/GEOPH.1991281849

Raikes, L., Straw, W., \& Linton, C. (2015). Total transport authorities a new deal for town and rural bus services. Institute for Public Policy Research. Recuperado de www.ippr.org

Rodríguez, V. (2011). Medición de la accesibilidad geográfica de la población a los Hospitales de Alta Resolución de Andalucía mediante herramientas SIG basadas en el análisis de redes. GeoFocus. Revista Internacional de Ciencia y Tecnología de La Información Geográfica, (11), 265-292. Recuperado de: https://www.geofocus.org/index.php/geofocus/article/view/220/72

Siabato, W., \& Guzmán-Manrique, J. (2019). La autocorrelación espacial y el desarrollo de la geografía cuantitativa. Cuadernos de Geografía: Revista Colombiana de Geografía, 28(1), 1-22. https://doi.org/10.15446/rcdg.v28n1.76919

Talen, E. (2001). School, Community, and Spatial Equity: An Empirical Investigation of Access to Elementary Schools in West Virginia. Annals of the Association of American Geographers, 91(3), 465 - 486. https://doi.org/10.1111/0004-5608.00254

Terigi, F., \& Shanno, C. (2013). Retos del Desarrollo Profesional Docente. Resumen Política. Recuperado de: https://www.oas.org/cotep/Library.aspx?lang=en\&iso=\&topic=\&category=\&keywor $\mathrm{d}=$ \&publicationyear=\&langs $=$ \&author=terigi\&Title $=$

UNESCO. (2013). Situación Educativa de América Latina y el Caribe: Hacia la educación de calidad para todos al 2015. Recuperado de:

http://www.unesco.org/new/fileadmin/MULTIMEDIA/FIELD/Santiago/images/SITI ED-espanol.pdf 